\title{
Investigation into the Influence of Fused Deposition Modeling (FDM) Process Parameters on the Thermal Properties of 3D-Printed Parts
}

\author{
Ahmed Elkholy and Roger Kempers ${ }^{1}$ \\ Department of Mechanical Engineering, \\ York University, \\ Toronto, Canada \\ 1 Corresponding Author: Kempers@yorku.ca
}

\begin{abstract}
The mechanical properties of 3D-printed parts have been extensively studied and the effects of printing parameters on them have been investigated. However, there are limited reliable data for the thermal properties of the materials used for printing, which can impede the development of additively manufactured heat exchangers made from either pure polymers or composites. In the current study, the effect of the layer height and width have been investigated experimentally and numerically to explore the thermal anisotropic nature of unidirectional printed parts printed using fused deposition modelling (FDM). The results show that increasing the layer height and width causes deterioration in the thermal conductivity, which may reach $65 \%$ of reduction compared to the pure polymer.
\end{abstract}

Keywords: FDM, FFF, printing parameters, thermal conductivty, PLA, numerical modelling

\section{INTRODUCTION}

Polymers have many advantages - such as corrosion resistance, low weight, and smooth surfaces - which make the class a notable competitor to exotic metals in heat exchanger applications. Nevertheless, their low thermal conductivity narrows their application [1]. The addition of conductive fillers inside the polymer matrix is an effective remedy to this issue. Polymer composites are conventionally produced by an injection process [2]. However, controlling the injection process parameters, such as injection flow conditions, filler volume concentration, their distribution, and their orientation state inside the polymer matrix, is not practically achievable.

Additive manufacturing (AM) is an alternative approach to produce composite polymer components [3], [4]. It depends on laying the material layer by layer according to the designed 3D CAD model. Compared with subtractive methods, it has many advantages, such as shortening the production time cycle and reducing cost [5], [6]. AM has many techniques, such as stereo-lithography (SLA), fused deposition modeling (FDM), selective laser sintering (SLS), and laminated object manufacturing (LOM) [7].
Recent studies show the ability to produce a final prototype of polymer heat exchangers using either one of the previous methods. For example, Jia et al. [8] employed the FDM process to produce a heat sink made of thermally conductive graphite polymer composite for a 3D printer extruder. Their results showed that it achieved a similar energy dissipation effectiveness compared with the conventional aluminum one. In the same direction, Hymas et al. [9] established a new hybrid approach of FDM and embedded metallic strips to fabricate a composite polymer heat exchanger (CPHE). Kalsoom et al. [10] exploited the stereolithography process to produce an electronic heat sink from composite resin made of synthetic diamond fillers and acrylate polymer. In this context, the current work is dedicated to studying the effect of the FDM process parameters on the thermal properties.

FDM is the most commonly used process parameter due to its ability to produce low cost products with negligible waste, the wide availability of various plastic filaments, and because there is no need for chemical post-processing. The process theory is based on laying the material layer by layer on a heated bed using a continuous filament of thermoplastic that passes through a hot nozzle moving in the $\mathrm{X}-\mathrm{Y}$ plane. The nozzle motion is controlled according to the data generated by slicing software which is responsible for dividing the CAD model into separate layers. Once one layer is completed, the bed is lowered in order to begin the other one and so on until a)

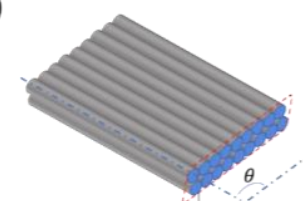

b)

b)

c)

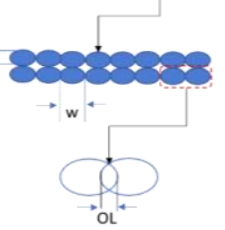

d)
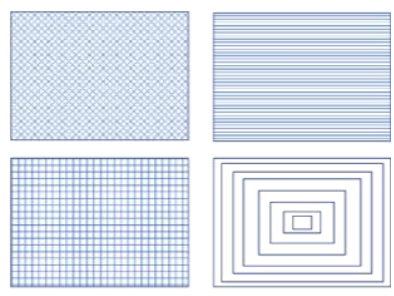

Figure 1: FDM process parameters; (a) raster angle, $\theta$; (b) layer height, $\mathrm{h}$ and width, w; (c) overlap, OL; (d) infill pattern. 
the whole product is built. This process has many parameters, such as layer height, layer width, overlap between layers, infill pattern, and raster angle, as shown in Fig. 1.

Due to the low mechanical and thermal properties of the FDM 3D-printed parts, many studies have focused on producing composite filament. However, this composite should have specific properties to be easily processed with FDM, such as viscosity, flexibility, stiffness, strength, and conductivity. Thus, for some cases where the filler concentration is high, additives - such as surfactants - are needed to produce homogeneous distribution of the filler inside the matrix. Some of these studies are presented here.

Nikzad et al. [11], [12] could increase the mechanical and thermal properties of Acrylonitrile Butadiene Styrene polymer (ABS) by including some metallic fillers-iron or copper-to produce a composite filament for FDM applications. They were able to successfully find the appropriate mixture percentage that meets the desired properties without facing processing issues during printing. They utilized the transient line source technique and the Differential Scanning Calorimetry (DSC) to measure thermal conductivity and the thermal capacity of the resulted mixture of composite polymer, respectively. Their results show the thermal conductivity achieved marked improvement above 30 vol \%. However, the thermal capacity deteriorated by incorporating the metallic fillers at any volume percentage. Furthermore, they examined the dynamic mechanical properties of the composite and demonstrated that high filler percentage reduced the material strength due to poor filler distribution, agglomeration, and the development of voids. Laureto et al. [13] used the guarded heat flow meter TCA300 to quantify the through-plane thermal conductivity of 3Dprinted parts made from the commercially available Polylactic acid (PLA) filament and its metal composites. Similar attempts were carried out, but with different metallic composites such as in the studies by Hwang et al. [14] and Masood et al. [15].

Ning et al. [2] examined experimentally the influence of process parameters such as nozzle temperature, raster angle, fill speed, and layer thickness on mechanical properties of carbon fiber reinforced polymers (CFRP). The adopted filament was made of an Acrylonitrile Butadiene Styrene (ABS) matrix with a volume concentration of $5 \%$ of chopped carbon fiber. They concluded that the optimum parameters are $220{ }^{\circ} \mathrm{C},[0 / 90], 25 \mathrm{~mm} / \mathrm{s}$ and 0.15 respectively in terms of the tensile properties. However, regarding the ductility and toughness, the optimum values remain the same, except for the layer thickness and raster angle which have values of $0.25 \mathrm{~mm}$ and [45/45], respectively. Furthermore, they investigated the effect of adding these carbon fibers on the porosity of the FDM-printed parts using an SEM micrograph. Shemelya et al. [16] utilized the Transient Plane Source (TPS) to address the anisotropic thermal properties of the 3D-printed ABS composites filled with graphite, carbon fiber, and silver. Flaata et al. [17] developed a steady-state apparatus designed specifically to measure the thermal conductivity of 3D-printed composites and exploited it to test some feed stock materials available commercially for FDM such as PLA, ABS, brass PLA, bronze PLA, and stainless steel PLA. However, there is a large discrepancy between the values measured by Laureto [13], Shemelya et al. [16], and Flaata et al. [17]. For instance, ABS has a thermal conductivity of 0.35 according to [17] while it was tested by [16] and suggested to have a value ranging from 0.15 to 0.2 , depending on the direction of measurements; this represents a deviation of $57 \%-75 \%$. The same situation applies for PLA and its composites. Also, some studies addressed the manufacture of piezoelectric ceramics and ceramic composites via FDM [10], [18], [19].

In summary, the mechanical properties of 3D-printed parts have been extensively studied and the effects of printing parameters on them have been investigated. However, to the best of our knowledge, there are limited studies about their thermal properties. The achievement of creating a thermally efficient composite polymer heat exchanger (CPHE) hangs on investigation of the anisotropic nature of the printed parts to produce reliable thermal conductivity data and exploration of the effects of process variables on their performance. Therefore, in order to achieve this goal, two objectives must be met:

- Develop an accurate experimental facility for measuring the thermal conductivity of low conductive materials.

- Characterize the effect of the layer height and width on the thermal conductivity of unidirectional printed parts experimentally and numerically.

\section{MATERIALS AND METHODOLOGY}

\section{A. Preparation of Samples}

To examine the effect of the process variables on the thermal anisotropic nature of unidirectional printed parts, the specimens need to be fabricated in three different configurations of the same size, as shown in Fig. 2. The first one is for quantifying the thermal conductivity in z-direction $\left(\mathrm{k}_{\mathrm{zz}}\right)$, while second one is for $\mathrm{y}$-direction $\left(\mathrm{k}_{\mathrm{yy}}\right)$ and the last one is for $\mathrm{x}$-direction $\left(k_{x x}\right)$. However, only the first direction was printed and measured experimentally, while the other directions were investigated numerically and analytically. The experimental measurement was utilized as a validation step for the numerical model. The influence of the layer height was studied by printing the samples in these configurations with

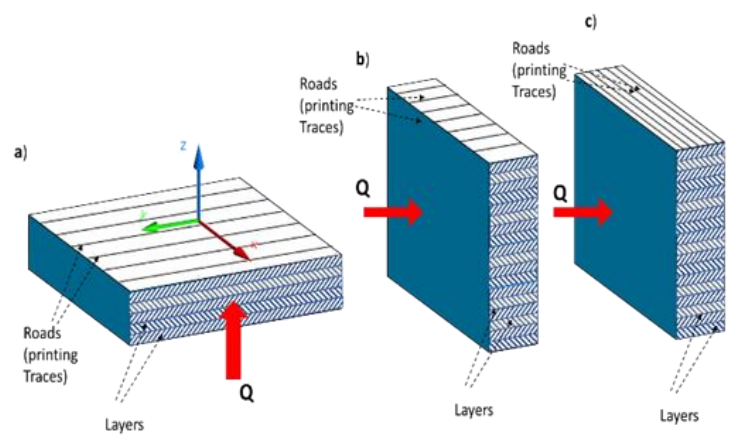

Figure 2: Different Configurations to measure the thermal conductivity in; (a) z-direction, $k_{z z}$, (b) y-direction, $k_{y y}$, (c) x-direction, $k_{x x}$. 
different values ranging from $0.05 \mathrm{~mm}$ to $0.3 \mathrm{~mm}$, while keeping the layer width and overlap constant at $0.4 \mathrm{~mm}$ and 0 $\mathrm{mm}$, respectively, which are the default printer setting. As for the layer width, its value was varied from $0.4 \mathrm{~mm}$ to $0.8 \mathrm{~mm}$, while maintaining the layer height and overlap at $0.15 \mathrm{~mm}$ and $0 \mathrm{~mm}$. The printing temperatures were $200{ }^{\circ} \mathrm{C}$ for the nozzle and $60{ }^{\circ} \mathrm{C}$ for the bed. All the samples were printed with line patterns with a raster angle of $90{ }^{\circ} \mathrm{C}$ in order to be unidirectional.

All samples were printed with $100 \%$ fill from PLA filament using the Ultimaker® 2 printer. Each sample was printed to the size $40 \mathrm{~mm} \times 40 \mathrm{~mm} \times 3 \mathrm{~mm}$, which is the same cross section area of the test rig. An automatic ULTRAPOL polishing machine was utilized to ensure that the sample surfaces were parallel and its surface roughness was very small. The sample surface roughness was measured after polishing by means of a profile meter and its Ra value was about $0.462 \mu \mathrm{m}$.

\section{B. Microscopic Study}

Determining the thermal conductivity for the other directions numerically or analytically requires an investigation of the pattern of layers after printing, which was carried out using a microscopic study. Also, this study was used to obtain the air volume fraction inside the matrix. Fig. 3a depicts the effect of changing the layer height from $0.15 \mathrm{~mm}$ to $0.3 \mathrm{~mm}$ while keeping its width at the default value of $0.4 \mathrm{~mm}$. Similarly, Fig. 3b shows the layer width effect while keeping the layer height at $0.15 \mathrm{~mm}$. As can be seen from this figure, increasing the layer height increases the air volume fraction and the same effect happens for the layer width. Fig. 3 indicates that the pattern is homogeneous, except for some roads which are not connected to each other. This phenomenon is more probable to occur at small layer heights. This is because the melt flow sometimes finds low resistance in one direction more than the other when squeezed against the printer bed. Another important feature which appears in this figure is that the roads starts to completely disconnect when layer width is more than $0.5 \mathrm{~mm}$. This additional air gap between the roads most probably happens because the printer is not able to provide the correct amount of extrusion during the printing time which is controlled by the travelling speed. The printing speed was held constant at a value of $60 \mathrm{~mm} / \mathrm{s}$ for all the samples. Each photo was then converted into an 8-bit image type in order to perform thresholding. ImageJ software was

a)

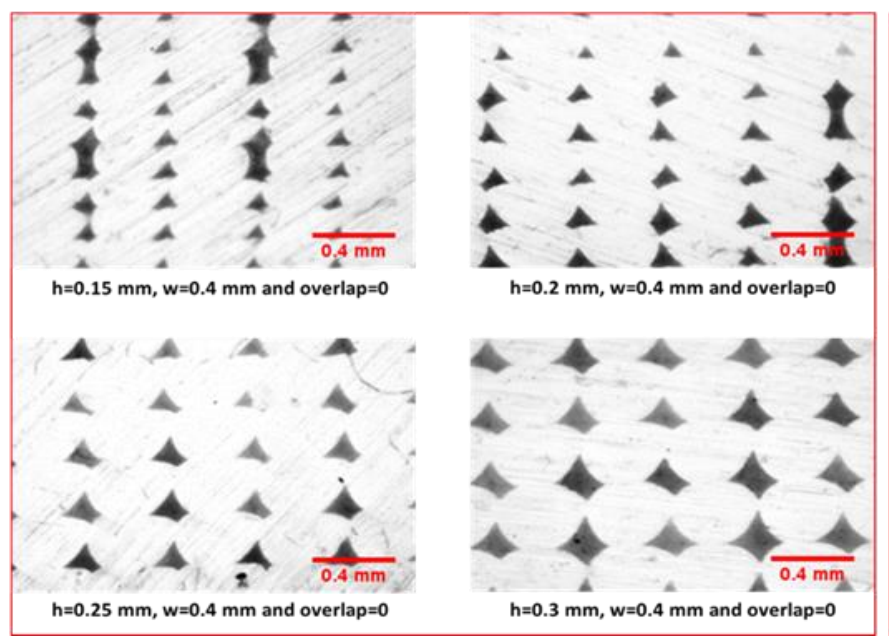

employed to do the thresholding and calculate the air volume fraction. Another method was employed to measure the volume fraction of air which depends on weighting the sample while postulating that the air mass inside the gaps is negligible, and then applying the following equation:

$$
\begin{aligned}
& v_{f}=\frac{v_{s}-v_{p}}{v_{s}} \\
& v_{p}=\frac{m_{s}}{\rho}
\end{aligned}
$$

where $v_{f}, v_{p}, v_{s}$ are the volume fraction of air, the volume that the polymer occupies inside the printed part, and the printed part volume. $\rho$ is the polymer density which was measured for the PLA feed stock filament. Table 1 and 2 show a comparison between the air volume fraction resulting from the ImageJ method and weighting method. As can be seen from these tables, there is a large deviation between the two methods, especially for the layer height. The reason for this is that the tested sample under the microscope is broken only at one section and the photo captured part of this section which cannot be considered representative of the whole sample, especially in given the layers' disconnection problem. Therefore, the second method was assumed to be more accurate; however, the photos give a better insight into the pattern happening inside the printed part and will help to develop the numerical model.

TABLE. I. AIR VOLUME FRACTION AT DIFFERENT LAYER HEIGHTS AT CONSTANT LAYER WIDTH OF 0.4 MM

\begin{tabular}{|c|c|c|c|}
\hline \multirow{2}{*}{$\begin{array}{c}\text { Layer } \\
\text { Height }\end{array}$} & \multicolumn{3}{|c|}{ Air Volume Fraction \% } \\
\cline { 2 - 4 } & ImageJ & \multicolumn{2}{|c|}{ Weighting Method } \\
\hline 0.1 & $v_{f} \%$ & $v_{f} \%$ & Uncertainty \% \\
\hline 0.15 & 4.1 & 8.2 & 0.14 \\
\hline 0.2 & 6.5 & 11.7 & 0.138 \\
\hline 0.25 & 9.8 & 13.5 & 0.13 \\
\hline
\end{tabular}

TABLE. II. AIR VOLUME FRACTION AT DIFFERENT LAYER WIDTHS AT CONSTANT LAYER HEIGHT OF 0.15 MM

\begin{tabular}{|c|c|c|c|}
\hline \multirow{2}{*}{$\begin{array}{c}\text { Layer } \\
\text { WIDTH, }\end{array}$} & \multicolumn{3}{|c|}{ Air Volume Fraction \% } \\
\cline { 2 - 4 } W & Image & \multicolumn{2}{|c|}{ Weighting Method } \\
\cline { 2 - 4 } & $v_{f} \%$ & $v_{f} \%$ & Uncertainty $\%$ \\
\hline 0.4 & 4.1 & 11.7 & 0.137 \\
\hline
\end{tabular}

b)

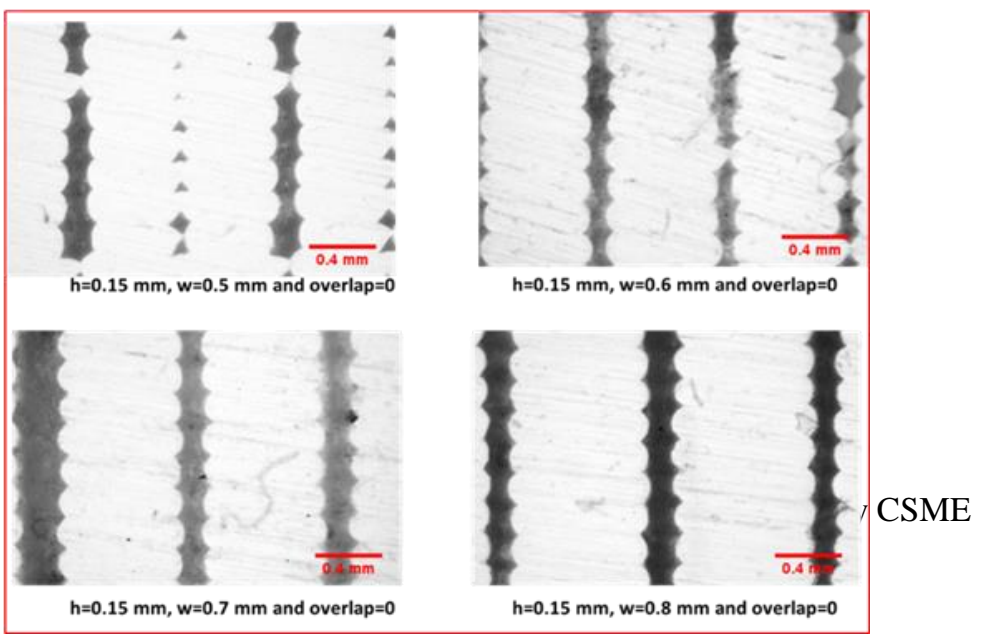




\begin{tabular}{|c|c|c|c|}
\hline \multirow{2}{*}{$\begin{array}{c}\text { Layer } \\
\text { WIDTH, }\end{array}$} & \multicolumn{3}{|c|}{ Air Volume Fraction \% } \\
\cline { 2 - 4 } & ImageJ & \multicolumn{2}{|c|}{ Weighting Method } \\
\hline 0.5 & 11.9 & 13.7 & 0.134 \\
\hline 0.6 & 13.7 & 15.3 & 0.131 \\
\hline 0.7 & 17.1 & 19 & 0.126 \\
\hline 0.8 & 18.1 & 24.5 & 0.117 \\
\hline
\end{tabular}

\section{Numerical Modeling}

The effective thermal conductivity in $\mathrm{z}$ or $\mathrm{x}$ directions can be predicted by simplifying the problem into a $2 \mathrm{D}$ model which resembles a unit cell that was extracted to represent the overall geometry. ANSYS fluent 18.2 software [20] was used to predict the heat flow by conduction through printed parts. The unit cell had a dimension of $h / 2$ in the $z$-direction and $w / 2$ in the x-direction, as shown in Fig. 4a. A temperature boundary condition was applied at the right and left boundaries of the domain, while insulated and symmetry conditions were adopted for the top and bottom boundaries, respectively, as shown in Fig. 4b. This configuration was employed to predict $k_{x x}$. As for the other direction, the boundary conditions were reversed, where the temperature gradient should be in the same direction as the thermal conductivity measurement direction. The energy equation solution results in the heat transfer which is used to calculate the effective thermal conductivity by exploiting Fourier's law of conduction. The simulation was repeated several times to find the layer height and width influence on the thermal properties. The same range stated in

a)

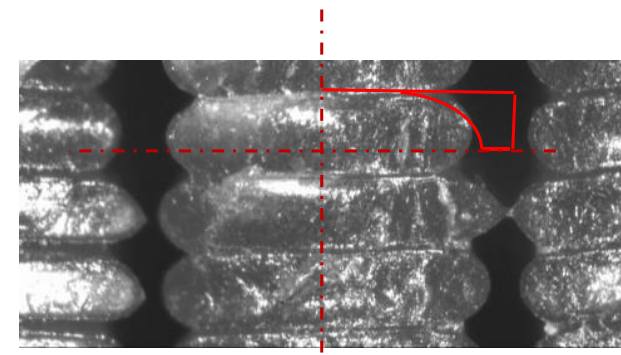

$\mathrm{h}=0.15 \mathrm{~mm}, \mathrm{w}=0.65 \mathrm{~mm}$ and $\mathrm{OL}=0$

b)

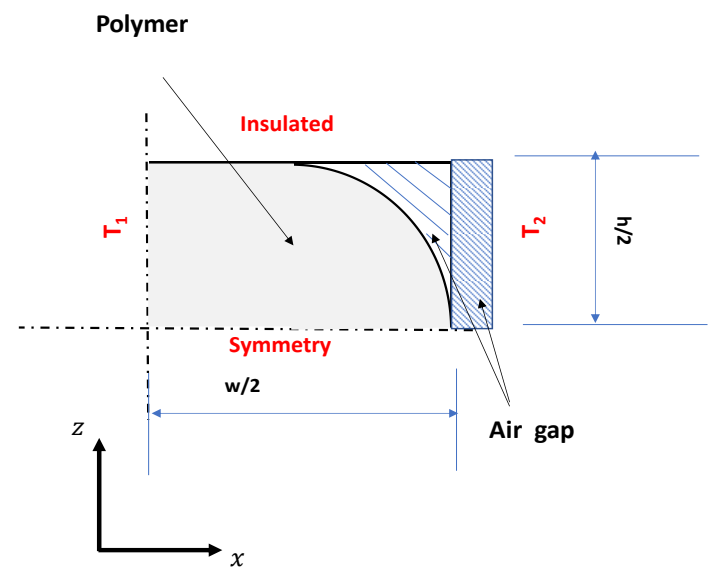

Figure 5: Numerical Model (a) unit Cell (b) domain.
Section A was used here for the numerical simulation. The employed temperature difference, $T_{1}-T_{2}$, was set equal to 1 for all cases. The volume fraction calculated by weighting the samples in the previous section was used to determine the air gap distance between the roads.

\section{Experimental Methodology}

Fig. 5 shows the suggested design which is mainly composed of two sides; one is serving as the heat source and the other one represents the heat sink, where the sample is sandwiched between them. The hot side is composed of two isothermal blocks manufactured from copper material, $\mathrm{k}=391.2 \mathrm{w} / \mathrm{m} \mathrm{k}$, with a small thickness to produce a uniform temperature field through them. The function of the first block is to supply the heat into the sample under testing. The secondary hot block was designed to surround the main one in order to guarantee that all the heat coming from the source flows into the sample only and there is no heat loss from the main block either from its sides or its top surface. The secondary block's power was controlled to ensure that the two blocks have the same temperature which was monitored at three different locations. It was assumed that the secondary power was adequately tuned when the temperature difference did not exceed $0.001{ }^{\circ} \mathrm{C}$ at steady state.

Similarly, the cold side consists of two blocks, which were also manufactured from copper material. Both have a $U$ channel which permits the chilled cooling water to flow through
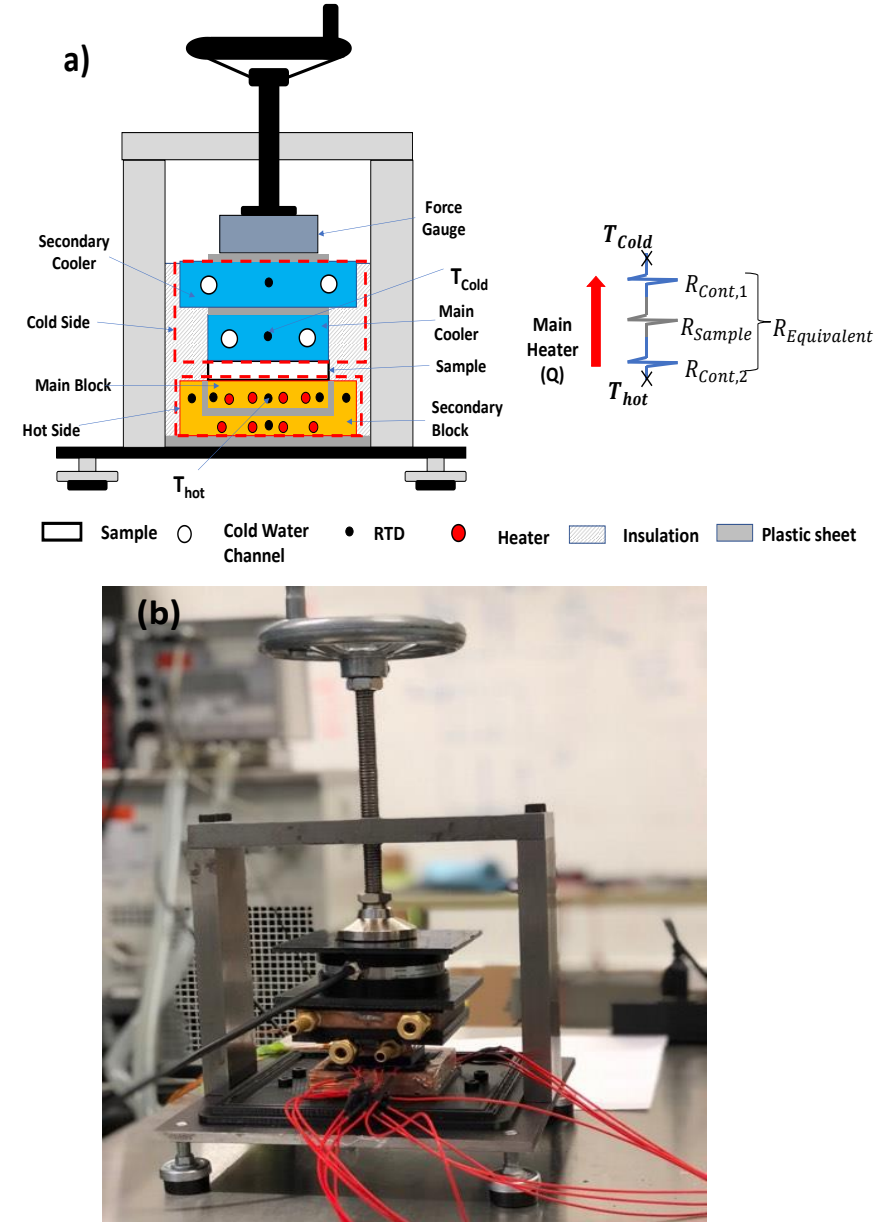

4 Figure 4: Experimental apparatus (a) Schematic (b) real rig 18 by CSME 
them. All the blocks and the sample were stacked above each other and forced against a force gauge using a clamping system. The secondary cold block function is to isolate the system from the clamping system base. All parts were confined into a large block filled with silica aerogel with a thermal conductivity of $0.014 \mathrm{w} / \mathrm{m} \mathrm{k}$.

The thermal conductivity can be easily calculated by measuring main block input power and temperature difference between RTD, as given by:

$$
k=\frac{Q \Delta t}{A_{c}\left(T_{\text {hot }}-T_{\text {cold }}\right)}
$$

where $k$ is the measured thermal conductivity, $\Delta t$ is the specimen thickness, $A_{c}$ is the sample cross section area and $\left(T_{h}-T_{c}\right)$ is the temperature difference between the main hot block and main cold one. Some aspects were considered while doing the testing to make sure that the contact resistance effect is negligible. Thermal interface material was applied at both sides of the sample and the clamping pressure was kept high at about 3 bars for all measurement. Moreover, the contact resistance value was calculated by testing the total thermal resistance for three samples, at the same operating conditions, with similar geometrical and printing parameters, except for the thickness which was different. The contact resistance value can be extracted by drawing its value against its thickness and performing a linear curve fitting. Its value was less than $2 \%$ of the total resistance, which can be discarded.

\section{RESULTS}

\section{A. The effect of layer height}

The thermal conductivity in z-direction $\left(k_{z z}\right)$ was measured experimentally and compared to the numerical model, as shown in Fig. 6. Each sample was measured three times and averaged to guarantee the reliability of the test rig. It is indicated that there is a good agreement between both of them which supports the possibility of using the numerical model to predict the thermal conductivity in $\mathrm{x}$-direction $\left(k_{x x}\right)$. The analytical parallel was employed to predict the thermal conductivity in the third direction $\left(k_{y y}\right)$ which is given by the following equation:

$$
K_{e}=\left(1-V_{f}\right) K_{m}+V_{f} K_{a}
$$

where $k_{e}$, is the effective thermal conductivity and $k_{m}$ and $k_{a}$ are the conductivities for the matrix and air respectively. $V_{f}$ is the air volume fraction inside the PLA matrix. The numerical model requires the thermal conductivity of pure polymer and this has been measured by printing a sample with a small layer height of $0.06 \mathrm{~mm}$ and a high percentage of overlap to ensure that there are no air gaps generated inside the part. Its value was $0.2207 \mathrm{w} / \mathrm{mk}$. The experimental results in Fig. 6 indicate that increasing the layer height reduces the thermal conductivity in $\mathrm{z}$-direction until a value of $0.133 \mathrm{w} / \mathrm{m} \mathrm{k}$ at layer height of $0.3 \mathrm{~mm}$ is reached, due to the increase of air volume fraction. This represents a percentage of reduction of about 40 $\%$ compared with pure PLA polymer. Figs. 6 and 7 illustrate that the effect of layer height is more significant in $\mathrm{z}$ and $\mathrm{x}$ directions, while it is nearly constant for y-direction. The thermal conductivities are higher in directions $\mathrm{z}$ and $\mathrm{y}$ than $\mathrm{x}$ direction. This is because the heat path in $\mathrm{x}$-direction is a series which increases the heat's overall resistance while it is parallel for the other directions. For $\mathrm{x}$-direction, the thermal conductivity decreases and then increases because when the layer height is small, the layers are not connected. In summary, the layer height increase causes a decrease in the thermal conductivity, which reaches values of reduction of $42 \%, 14 \%$, and $28 \%$ for $\mathrm{x}, \mathrm{y}$, and $\mathrm{z}$, respectively, compared with the pure polymer at a layer height of $0.3 \mathrm{~mm}$. The previous percentages were calculated based on the numerical model predictions.

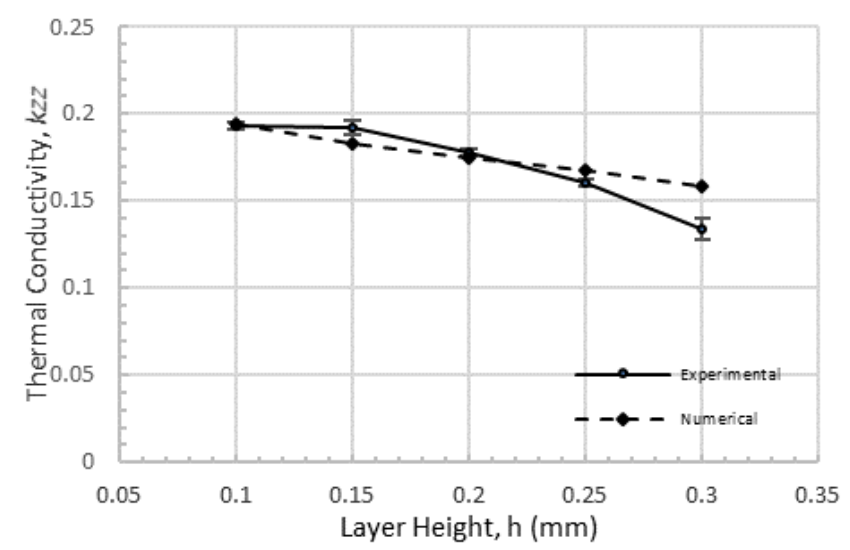

Figure 6: Comparison between the experimental results and numerical model for studying the effect of layer height on the thermal conductivity, $k_{z z}$ at constant layer width of $0.4 \mathrm{~mm}$.

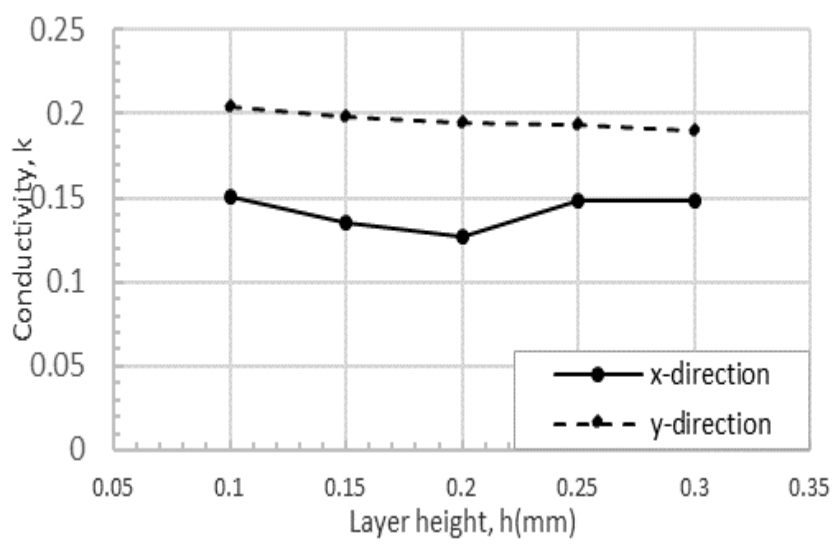

Figure 7: The effect of layer height on the thermal conductivity in y-direction $\left(k_{y y}\right)$ and $\mathrm{x}$-direction $\left(k_{x x}\right)$ at constant layer height of $0.15 \mathrm{~mm}$.

\section{B. The effect of layer width}

The influence of the layer width on thermal conductivity in $\mathrm{x}$ and $\mathrm{z}$ directions was predicted numerically while the parallel model was employed for y-direction, as shown in Fig. 8. Similar to the layer height, increasing the layer width causes 
deterioration for the thermal conductivity in all directions. Also, the thermal conductivity is higher for $\mathrm{y}$ and $\mathrm{z}$ directions than $\mathrm{x}$-direction, because of the same reason mentioned before. The layer width has a severe effect on the thermal conductivity in x-direction because the layers are completely disconnected in that direction.

The thermal conductivity in $\mathrm{x}$-direction reaches a low thermal conductivity value of $0.077 \mathrm{w} / \mathrm{mk}$ at a width of 0.8 $\mathrm{mm}$, which represents a $65 \%$ reduction compared with pure polymer, while the reduction is $28 \%$ and $25 \%$ for $\mathrm{y}$ and $\mathrm{z}$ directions, respectively. This highlights the importance of studying these parameters and demonstrates the possibility of tailoring the thermal properties of the printed parts, especially in the case of continuous fiber printing.

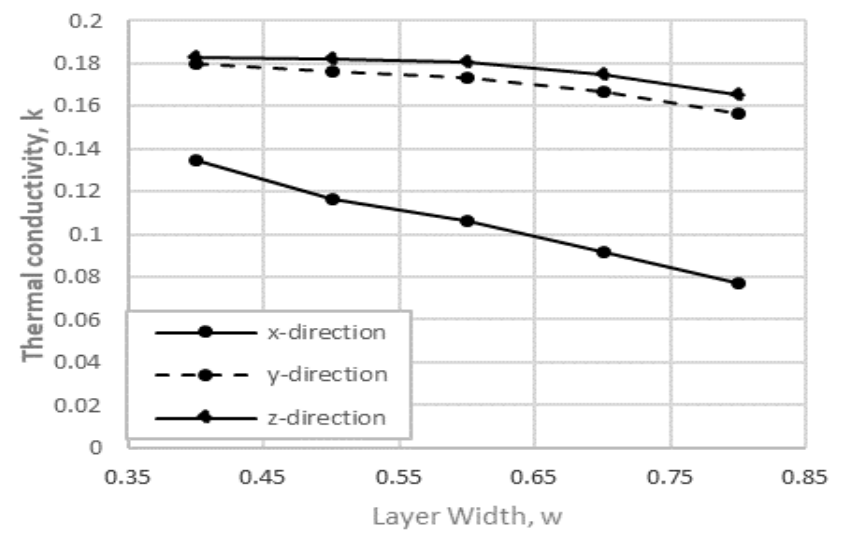

Figure 8: The effect of layer width on the thermal anisotropic nature of 3Dprinted parts.

\section{CONCLUSION}

This work examined the influence FDM process parameters on the anisotropic thermal properties of unidirectional 3D-printed parts. An experimental facility was developed to measure the effect of the layer height on the thermal conductivity in one direction, which was used as a validation step for the developed numerical model. The numerical model used in parallel with the analytical model was able to quantify the thermal conductivities in all directions. The main outcomes can be summarized into these points:

- Increasing either the layer height or width reduces thermal conductivity in all directions as a result of porosity generation.

- The effect of layer width is more significant than the layer height, especially when the layer disconnection problem exists.

- Increasing the layer height, while keeping the layer width constant at $0.4 \mathrm{~mm}$, results in a drop in the thermal conductivity which reaches a value of $42 \%$, $14 \%$, and $28 \%$ in $\mathrm{x}, \mathrm{y}$, and $\mathrm{z}$ directions, respectively, at a layer height of $0.3 \mathrm{~mm}$ compared with pure polymer.

- Increasing the layer width, at constant layer height of 0.15 , leads to a decline in thermal conductivity, reaching reduction percentages of $65 \%, 28 \%$, and
$25 \%$ in $\mathrm{x}, \mathrm{y}$, and $\mathrm{z}$ directions, respectively, at a layer width of $0.8 \mathrm{~mm}$.

\section{REFERENCES}

[1] I. L. Ngo, S. Jeon, and C. Byon, "Thermal conductivity of transparent and flexible polymers containing fillers: A literature review," International Journal of Heat and Mass Transfer. 2016.

[2] F. Ning, W. Cong, Y. Hu, and H. Wang, "Additive manufacturing of carbon fiber-reinforced plastic composites using fused deposition modeling: Effects of process parameters on tensile properties," $J$. Compos. Mater., vol. 51, no. 4, pp. 451-462, 2017.

[3] S. Kumar and J. P. Kruth, "Composites by rapid prototyping technology," Mater. Des., 2010.

[4] A. C. De Leon, Q. Chen, N. B. Palaganas, J. O. Palaganas, J. Manapat, and R. C. Advincula, "High performance polymer nanocomposites for additive manufacturing applications," Reactive and Functional Polymers. 2016

[5] B. Berman and F. G. Zarb, "3-D printing: The new industrial revolution 1. A multi-faceted technology: 3-D printing," Bus. Horiz., vol. 55, pp. 155-162, 2011.

[10] U. Kalsoom, A. Peristyy, P. N. Nesterenko, and B. Paull, "A 3D printable diamond polymer composite: a novel material for fabrication of low cost thermally conducting devices."

[11] M. Nikzad, S. H. Masood, and I. Sbarski, "Thermo-mechanical properties of a highly filled polymeric composites for Fused Deposition Modeling," Mater. Des., 2011.

[12] M. Nikzad, S. H. Masood, I. Sbarski, and a Groth, "ThermoMechanical Properties of a Metal-filled Polymer Composite for Fused Deposition Modelling Applications," 5th Australas. Congr. Appl. Mech. ACAM 2007, vol. 1, no. December, pp. 319-324, 2007.

[13] J. Laureto, J. Tomasi, J. A. King, and J. M. Pearce, "Thermal properties of 3-D printed polylactic acid-metal composites," Prog. Addit. Manuf., vol. 2, no. 1-2, pp. 57-71, 2017.

[14] S. Hwang, E. I. Reyes, K. sik Moon, R. C. Rumpf, and N. S. Kim, "Thermo-mechanical Characterization of Metal/Polymer Composite Filaments and Printing Parameter Study for Fused Deposition Modeling in the 3D Printing Process," J. Electron. Mater., 2015.

[15] S. H. Masood and W. Q. Song, "Assembly Automation Thermal characteristics of a new metal/polymer material for FDM rapid prototyping process Thermal characteristics of a new metal/polymer 
material for FDM rapid prototyping process," Assem. Autom. Rapid Prototyp. J. Iss Rapid Prototyp. J., vol. 25, no. 11, pp. 309-315, 2002.

[16] C. Shemelya, A. De La Rosa, A. R. Torrado, K. Yu, J. Domanowski, P. J. Bonacuse, R. E. Martin, M. Juhasz, F. Hurwitz, R. B. Wicker, B. Conner, E. MacDonald, and D. A. Roberson, "Anisotropy of thermal conductivity in 3D printed polymer matrix composites for space based cube satellites," Addit. Manuf., vol. 16, pp. 186-196, 2017.

[17] T. Letcher, "Thermal Conductivity Testing Apparatus for 3D Printed Materials THERMAL CONDUCTIVITY TESTING
APPARATUS FOR 3D PRINTED MATERIALS,” no. July, 2017.

[18] M. K. Agarwala, R. Van Weeren, A. Bandyopadhyay, P. J. Whalen, A. Safari, and S. C. Danforth, "Fused deposition of ceramics and metals: an overview," Proc. Solid Free. Fabr. Symp., pp. 385-392, 1996.

[19] A. Bandyopadhyay, R. K. Panda, T. F. McNulty, F. Mohammadi, S. C. Danforth, and A. Safari, "Piezoelectric ceramics and composites via rapid prototyping techniques," Rapid Prototyp. J., vol. 4, no. 1, pp. 37-49, 1998.

[20] ANSYS, “ANSYS Fluent Theory Guide," ANSYS 16 Documentation, vol. 15317, no. July. p. 80, 2015. 